\title{
Low ATM expression and progression-free and overall survival in advanced gastric cancer patients treated with first-line XELOX chemotherapy
}

\author{
Samuel J. Klempner ${ }^{1,2 \#}$, Munveer S. Bhangoo ${ }^{3 \#}$, Hubert Y. Luu ${ }^{4}$, Seung Tae Kim ${ }^{5}$, Joseph Chao ${ }^{6}$, Kyoung- \\ Mee $\mathrm{Kim}^{7}$, Jeeyun Lee \\ ${ }^{1}$ The Angeles Clinic and Research Institute, Los Angeles, CA, USA; ${ }^{2}$ Samuel Oschin Comprehensive Cancer Institute, Cedars-Sinai Medical Center, \\ Los Angeles, CA, USA; ${ }^{3}$ Division of Hematology Oncology, Scripps Clinic, La Jolla, CA, USA; ${ }^{4}$ Department of Surgery, University of California, \\ San Francisco, CA, USA; ${ }^{5}$ Department of Medicine, Samsung Medical Center, Seoul, Korea; ${ }^{6}$ Department of Medical Oncology and Therapeutics \\ Research, City of Hope Comprehensive Cancer Center, Duarte, CA, USA; ${ }^{7}$ Department of Pathology and Translational Genomics, Samsung \\ Medical Center, Seoul, Korea \\ Contributions: (I) Conception and design: SJ Klempner, MS Bhangoo, J Lee; (II) Administrative support: J Lee; (III) Provision of study materials or \\ patients: ST Kim, KM Kim; (IV) Collection and assembly of data: All authors; (V) Data analysis and interpretation: All authors; (VI) Manuscript \\ writing: All authors; (VII) Final approval of manuscript: All authors. \\ \#These authors contributed equally to this work. \\ Correspondence to: Samuel J. Klempner, MD. Director of Precision Medicine and GI Oncology, The Angeles Clinic and Research Institute, Cedars- \\ Sinai Medical Center, Los Angeles, CA, USA. Email: sklempner@theangelesclinic.org.
}

Background: Gastric cancer (GC) is a leading cause of cancer-specific mortality with limited biologically informed treatments. The ataxia telangiectasia mutated $(A T M)$ gene is critically involved in the repair of double-stranded DNA breaks and a component of DNA damage repair (DDR) pathways. Platinum salts are hypothesized to have increased efficacy in tumors deficient in DDR pathways. We sought to investigate an association between ATM status and response to XELOX in a homogenous first line GC patient cohort.

Methods: A clinically annotated cohort of 137 Korean patients with advanced GC treated with first-line XELOX was retrospectively examined for ATM status by immunohistochemistry. Correlation between ATM expression and clinicopathologic variables was performed by two-tailed, unpaired $t$-tests and Fisher's exact tests. Kaplan-Meier survival analysis curves and Cox proportional hazards models were used to evaluate for independent predictors of disease-free survival (DFS) and overall survival (OS).

Results: Low ATM expression was observed in 19.0\% (26/137) of patients and was not associated with clinicopathologic features or response rate to XELOX. Univariate, but not multivariable, logistic regression and Cox analysis identified ATM as an independent risk factor influencing OS and DFS. A higher ECOG score independently predicted worse survival [hazard ratio (HR) 2.96, $\mathrm{P}=0.016$ ] and complete surgical resection independently protected against progression of disease (HR 0.69, $\mathrm{P}=0.007$ ).

Conclusions: Low ATM expression was not associated with increased response rates to XELOX in a single-institution cohort of advanced GC patients. Similarly, ATM status did not predict DFS or OS after platinum-based chemotherapy.

Keywords: Stomach cancer; ataxia telangiectasia mutated (ATM); DNA damage; PARP; platinum

Submitted Jul 08, 2018. Accepted for publication Aug 14, 2018.

doi: 10.21037/jgo.2018.09.04

View this article at: http://dx.doi.org/10.21037/jgo.2018.09.04 


\section{Introduction}

Gastric adenocarcinoma is a leading cause of cancerrelated death globally (1). Although considerable progress has been made in the molecular classification of gastric cancer (GC), the prognosis remains poor with overall survival (OS) ranging from 10 to 16 months in metastatic disease (2-5). Evidence-based targeted approaches based on specific molecular profiles are limited to HER2 amplified and a small subset of microsatellite instable (MSI)-high tumors (6). There exists considerable unmet need for predictive biomarkers to guide the rational use of existing therapies and to identify novel therapeutic approaches or combinations.

Acquired defects in DNA damage repair (DDR) pathways are a defining mechanism of tumorigenesis (7). Both germline and somatic mutations in DDR genes are not only associated with increased risk of the development of cancer but also represent attractive targets for therapeutic intervention. The ataxia telangiectasia mutated (ATM) gene is critically involved in the repair of double-stranded DNA breaks. Specifically, ATM localizes to sites of doublestranded DNA breaks and interacts with BRCA1 (breast cancer type 1) and 53BP1 (p53-binding protein) (8). ATM affects multiple downstream pathways activating DNAdamage repair along with cell-cycle arrest and apoptosis (9).

Platinum salts are hypothesized to have increased efficacy in DDR-deficient tumors. Mechanistically, platinum drugs induce double-strand DNA breaks which selectively leads to unsustainable levels of genomic instability resulting in tumor cell death. In DDR-defective cells the impaired repair machinery is believed to underlie the synthetic lethality after treatment with platinum compounds. Patients with BRCA1- and BRCA2-mutated breast, ovarian, and prostate cancer appear to exhibit increased responsiveness to platinum-based chemotherapy (10).

Low expression of ATM in GC is associated with poorlydifferentiated histology, lymph node involvement, and worse overall 5 -year survival among patients undergoing curative surgical resection (11). Pre-clinical models have suggested increased sensitivity of ATM deficient cells to inhibition with olaparib and SN-38 though platinum associations remain understudied (12). Recently, the phase III GOLD trial failed to show a survival benefit for olaparib in unselected advanced GC progressing after first-line therapy. This lack of benefit was seen in both the overall study population as well as patients with tumors expressing low levels of ATM (13).
Whether or not deficiencies in ATM are associated with increased tumor response and survival in patients with GC treated with platinum chemotherapy is currently unknown. Identifying molecularly defined GC patient subgroups who are more, or less, likely to benefit from a given approach is an ongoing need in GC. We sought to explore the relationship between outcomes and ATM status in a homogenous cohort of Korean patients with advanced GC treated with first-line capecitabine in combination with oxaliplatin (XELOX).

\section{Methods}

\section{Patients}

We retrospectively reviewed patients with advanced GC (unresectable stage 3 or stage 4) at Samsung Medical Center (Seoul, Korea) from 2013 to 2017. All patients received first-line (1L) capecitabine and oxaliplatin (XELOX) at standard dosing. Data was abstracted from medical records including sex, age at diagnosis, performance status. The study was approved by the institutional review board at Samsung Medical Center and all data was stored securely (2015-10-062). Pathologic features including pathologic subtype, anatomic site, and HER2/neu status were collected. Outcomes data included tumor response, and the date of progression or death.

\section{Immunobistochemistry (IHC)}

Tumor sections ( $2 \mathrm{~mm}$ in diameter) were obtained from formalin-fixed paraffin-embedded biopsy samples. Tissue was arranged in new recipient paraffin block (tissue array block) utilizing a trephine apparatus (Superbiochips Laboratories, Seoul, Korea) as previously reported (14). Immunohistochemical staining was performed using an automatic immunostainer (DAKO, Glostrup, Denmark) according to the manufacturer's instructions. The primary antibody used was anti-ATM antibody [Y170]. Lowexpression of ATM $\left(\mathrm{ATM}_{\mathrm{low}}\right)$ was defined as greater than $50 \%$ loss of nuclear staining.

\section{Statistics}

Descriptive statistics were reported as proportions and means. Correlation between ATM expression and clinicopathologic variables were performed by two-tailed, unpaired $t$-tests and Fisher's exact tests. Treatment outcomes 
included overall response rate (ORR), progression-free survival (PFS), and OS. PFS was defined as the date of first chemotherapy to the date of cancer progression, death from any cause or the date of last follow-up visit. OS was defined as the date of first chemotherapy to the date of death from any cause and was censored at the date of the last followup visit. Independent variables were selected a priori for analysis in multivariable logistic regression. Kaplan-Meier survival analysis was used to compare disease-free survival (DFS) and OS; the log-rank test was used to determine statistical significance. Cox proportional hazards modeling was used to assess the impact of individual risk factors on length of survival or time to progression of disease. Variables with observed $\mathrm{P}$ values $<0.05$ were considered statistically significant for this analysis, and $95 \%$ CI were calculated. All statistical analyses were performed using STATA 15.0 (STATACorp LLC, College Station, Texas, USA).

\section{Results}

\section{Clinicopathologic features}

Baseline characteristics $(\mathrm{n}=137)$ are presented in Table 1 . The mean age of all patients was 55.7 years (range, 26-80 years). Fifty-one patients $(37.2 \%)$ were female. The most common histologic subtypes by WHO classification were poorlydifferentiated adenocarcinoma $(n=68,49.6 \%)$, signet ring adenocarcinoma $(n=29,21.2 \%)$, and moderately differentiated adenocarcinoma $(\mathrm{n}=28,20.4 \%)$. The primary tumors include gastric body $(\mathrm{n}=73,53.3 \%)$, antrum $(\mathrm{n}=41$, $29.9 \%)$, cardia $(n=16,11.7 \%)$, other $(n=6,4.4 \%)$. Onehundred and thirty-two (96.4\%) patients had ECOG status of 0 or 1 . HER2/neu overexpression was absent in 130/135 patients $(96.3 \%)$ (Table 1$)$.

\section{Outcomes of patients with $A T M_{\text {loss }}$ vs. $A T M_{\text {intact }}$}

Among the 137 patients analyzed for ATM expression, 26 $(19.0 \%)$ exhibited low or undetectable ATM expression by IHC (Figure 1, Table 1). Unadjusted univariate analysis demonstrated an association between $\mathrm{ATM}_{\text {low }}$ status and ECOG status $(\mathrm{P}=0.046)$ and prior surgery $(\mathrm{P}=0.041)$. All other clinicopathologic variables showed no association with ATM expression. Multivariable logistic regression was performed including the variables age, sex, ECOG status, WHO classification, HER2 status, performance of surgical resection, primary tumor location, stage, and ATM status. Advanced ECOG status was independently associated with worse OS [odds ratio (OR) 4.83; 95\% CI: 1.58-14.78, $\mathrm{P}=0.006$, Table 2]. This was also seen on Kaplan-Meier survival analysis (Figure S1).

\section{Oncologic response to XELOX based on ATM expression}

All patients analyzed received capecitabine plus oxaliplatin as $1 \mathrm{~L}$ systemic therapy for advanced gastric adenocarcinoma. The mean number of cycles received was 7.1 (range, 1-23). The ORR across the entire cohort was $31.4 \%$, composed entirely of partial responses (Table 3). The ORR for ATM $\mathrm{Amw}_{\text {low }}$ was $26.9 \%$ compared with $32.4 \%$ in among patients with $\mathrm{ATM}_{\text {intact }}$ status, not statistically significant $(\mathrm{P}=0.800)$. The median PFS for the overall study population was 6.12 months (Figure $2 A$ ), consistent with prior $1 \mathrm{~L}$ trials (2). The median OS for the study population was 10.1 months (Figure 2B). Among all 137 patients, 53 deaths (39\%) occurred in the follow-up period. Crude analysis based on Kaplan-Meier curves showed no difference in survival outcomes for patients with $\mathrm{ATM}_{\text {low }}$ versus $\mathrm{ATM}_{\text {intact }}$ patients in both OS and PFS (Figure 2C,D).

\section{Univariate and multivariable Cox models}

In our univariate Cox model patients with an ECOG higher than 1 had significantly increased risk of death compared to those with ECOG 1 or lower. Patients who underwent a curative surgical resection (either total or subtotal gastrectomy) had significantly decreased risk of recurrence over the analysis period. These findings were robust in multivariable Cox analysis both ECOG [hazard ratio (HR) 2.80; $\mathrm{P}=0.02,95 \%$ CI: $1.19-6.60$, Table 4] and for prior curative resection (HR 0.69; $\mathrm{P}=0.007,95 \%$ CI: $0.52-0.90$, Table 4). ATM status was not significantly associated with OS or PFS in the multivariable-adjusted Cox model.

\section{Discussion}

Deficient ATM expression was identified in 1/5 of advanced Korean GC patients but was not associated with improved outcomes with 1L XELOX. The incidence of low or absent ATM expression in our population is consistent with prior reports. A large retrospective cohort of ATM expression in GC tumors revealed low ATM expression in $22 \%$ of cases, consistent between primary and metastatic tumor samples (15).

Deleterious genomic alterations involving ATM occur in 10 percent of GC (16). The discordance in the frequency of gene alterations compared with protein expression (as 
Table 1 Baseline characteristics of 137 Korean patients with advanced GC treated with first-line XELOX

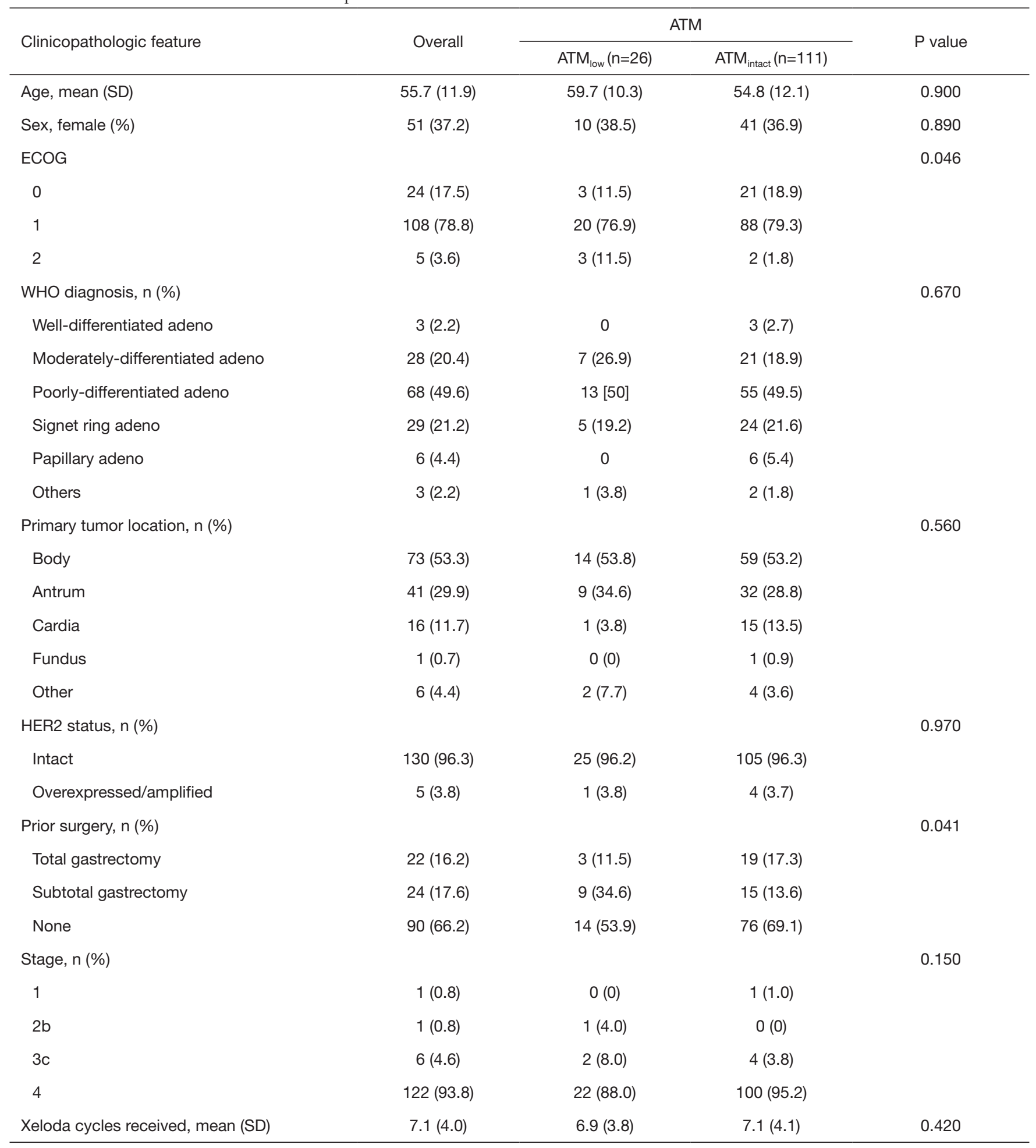

GC, gastric cancer; XELOX, capecitabine in combination with oxaliplatin. 

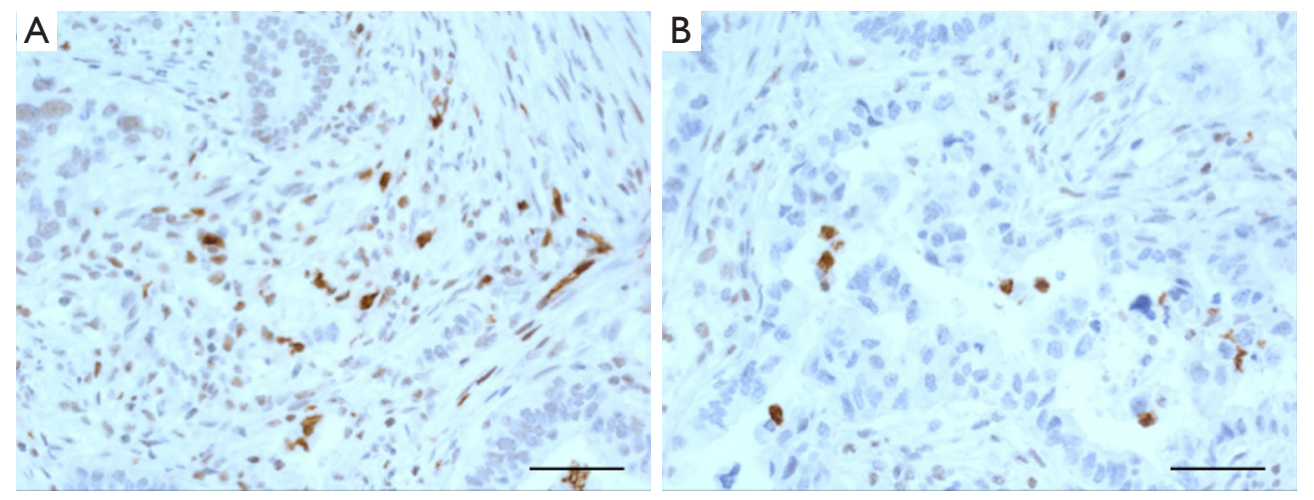

Figure 1 Representative IHC demonstrating ATM intact (A) and ATM loss in 70\% tumor cells (B) at 400× magnification (scale bar =50 $\mu \mathrm{m}$ ).

Table 2 Multivariable logistic regression analysis in a cohort of GC patients treated with $1 \mathrm{~L}$ XELOX

\begin{tabular}{lccc}
\hline Variables & OR & $95 \% \mathrm{Cl}$ & P value \\
\hline Age & 1.01 & $0.98-1.05$ & 0.450 \\
Sex & 1.10 & $0.47-2.57$ & 0.820 \\
ECOG & 4.83 & $1.58-14.78$ & 0.006 \\
WHO & 0.93 & $0.67-1.29$ & 0.660 \\
HER2 status & 1.21 & $0.18-8.02$ & 0.840 \\
Surgical resection & 0.94 & $0.55-1.59$ & 0.810 \\
Tumor location & 1.05 & $0.72-1.52$ & 0.800 \\
Stage & 1.04 & $0.37-2.94$ & 0.950 \\
ATM status & 0.47 & $0.16-1.35$ & 0.160 \\
\hline
\end{tabular}

GC, gastric cancer; OR, odds ratio; 1L, first line; XELOX, capecitabine in combination with oxaliplatin; ATM, ataxia telangiectasia mutated.

measured by IHC) may be a consequence of non-genomic changes affecting protein expression. Our approach was not oriented to study the genomic context of ATM loss though further investigation is warranted, however, the apparent mutual exclusivity of ATM loss and HER2 amplification from TCGA data is noted and confirmed in our cohort.

The data presented here represent the first report of $1 \mathrm{~L}$ outcomes in advanced GC patients treated with platinumchemotherapy and stratified by ATM expression. In this cohort, no difference was observed in terms of response rates to platinum-based chemotherapy between $\mathrm{ATM}_{\text {low }}$ and $\mathrm{ATM}_{\text {intact }}$ patients. Our results may be somewhat confounded by the uniform Asian population which tends toward improved outcomes in several prior trial datasets (17).
Table 3 Response characteristics of 137 Korean patients treated with XELOX and assessed for ATM expression

\begin{tabular}{lccc}
\hline Clinical outcome & ATM $_{\text {low }}, \mathrm{n}(\%)$ & ATM $_{\text {intact }}, \mathrm{n}(\%)$ & $\mathrm{P}$ value \\
\hline Complete response & 0 & 0 & 0.8 \\
Partial response & $7(26.9)$ & $36(32.4)$ & \\
Stable disease & $15(57.7)$ & $62(55.9)$ & \\
Progressive disease & $4(15.4)$ & $13(11.7)$ & \\
Disease control rate & $22(84.6)$ & $98(88.3)$ & \\
\hline
\end{tabular}

XELOX, capecitabine in combination with oxaliplatin; ATM, ataxia telangiectasia mutated.

However, the homogenous population (single Korean institution) studied here may be considered both a strength and weakness, and external validation in non-Asian patients is important. While we acknowledge the relatively small sample size to our knowledge this remains the largest reported cohort. The retrospective nature of this analysis also is an inherent limitation.

The clinical significance of aberrant homologous recombination repair pathways defined by genomic alteration or deficient protein expression in predicting response to platinum-based chemotherapy is an area of ongoing clinical interest. Pre-clinical models have demonstrated the importance of both BRCA1 and BRCA2 in assembly of the Rad51 complex which is essential to the repair of double-stranded DNA breaks $(18,19)$. Patients with BRCA-associated ovarian cancer demonstrate improved survival versus those non-BRCA mutated tumors. One mechanism for improved outcomes may be increased sensitivity to platinum-based chemotherapy $(10,20)$. Similar associations between alterations in BRCA1 and BRCA2 and 

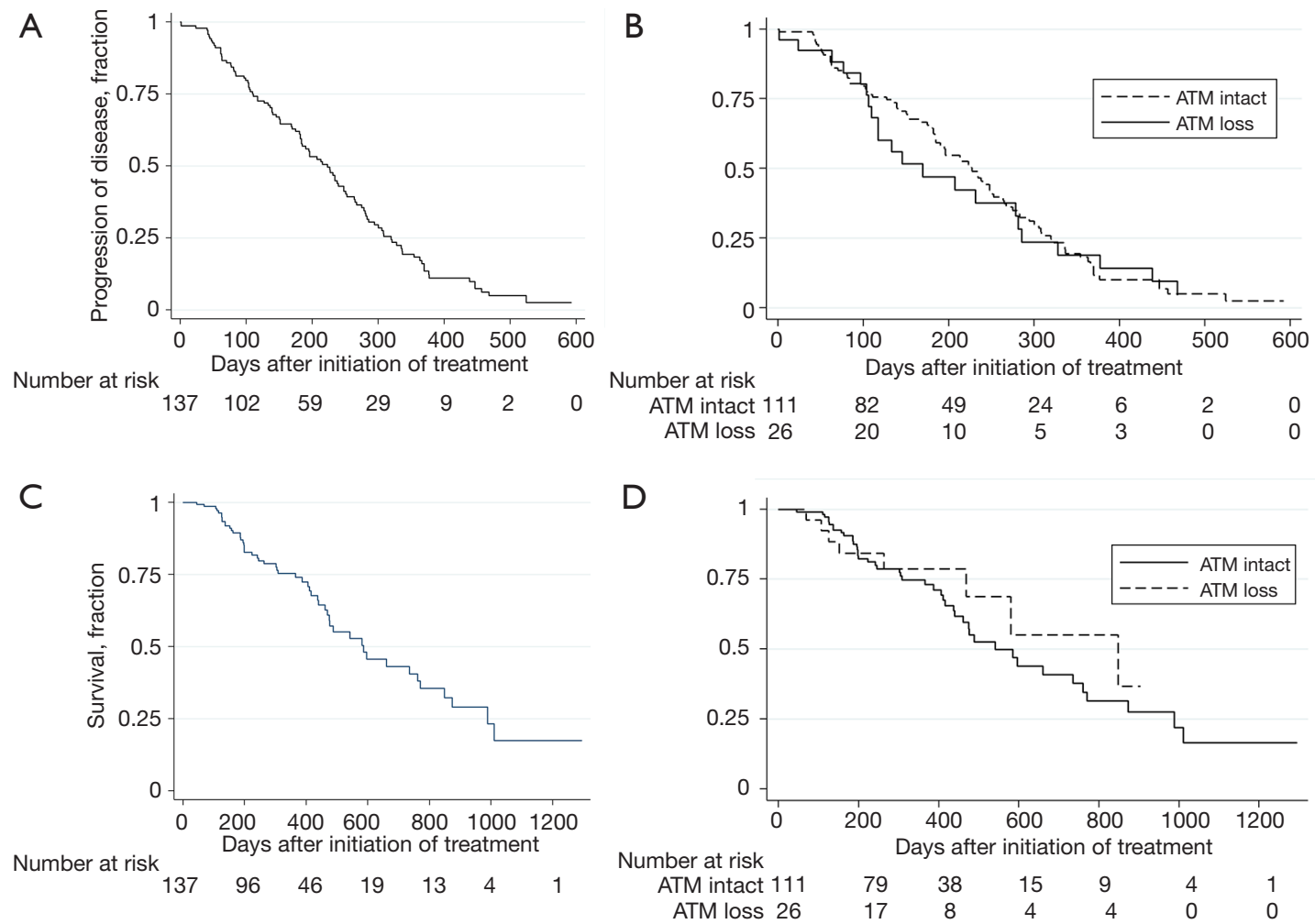

Figure 2 GC outcomes with 1L XELOX. (A,B) Progression-free survival for all patients (A) and stratified by ATM status (B); (C,D) overall survival for all patients (C) and stratified by ATM status (D). GC, gastric cancer; 1L, first line; ATM, ataxia telangiectasia mutated; XELOX, capecitabine in combination with oxaliplatin.

Table 4 Multivariate Cox proportional hazard analysis of OS and PFS in a cohort of GC patients treated with 1L XELOX and assessed for ATM expression

\begin{tabular}{|c|c|c|c|c|c|c|}
\hline Variables & \multicolumn{3}{|c|}{ Overall survival } & \multicolumn{3}{|c|}{ Progression-free survival } \\
\hline Age & 0.99 & $0.97-1.02$ & 0.980 & 0.99 & $0.98-1.02$ & 0.990 \\
\hline Sex & 1.05 & $0.56-1.94$ & 0.890 & 0.92 & $0.57-1.48$ & 0.740 \\
\hline ECOG & 2.80 & $1.19-6.60$ & 0.019 & 1.49 & $0.90-2.45$ & 0.120 \\
\hline HER2 & 1.69 & $0.40-7.21$ & 0.480 & 0.39 & $0.14-1.12$ & 0.080 \\
\hline Surgical resection & 1.14 & $0.78-1.67$ & 0.510 & 0.69 & $0.52-0.90$ & 0.007 \\
\hline ATM status & 0.66 & $0.30-1.47$ & 0.310 & 1.49 & $0.88-2.50$ & 0.140 \\
\hline
\end{tabular}

OS, overall survival; PFS, progression-free survival; 1L, first line; GC, gastric cancer; XELOX, capecitabine in combination with oxaliplatin; ATM, ataxia telangiectasia mutated. 
platinum-sensitivity are seen in breast and prostate cancer $(10,21,22)$.

Mechanistically, alterations in ATM are predicted to interfere with homologous recombination repair and, therefore, potentially serve as a predictive biomarker for DNA-damaging therapies. In pre-clinical models, the significance of ATM defects has been variably demonstrated to be associated with increased sensitivity to platinum chemotherapy. One study of ATM-deficiency in mammary cells demonstrated increased sensitivity to DNA-damaging agents (23). Yet, two other pre-clinical reports have failed to consistently demonstrate the significance of ATM in predicting platinum-sensitivity $(24,25)$. Mutations in genes involved in homologous recombination repair including $B R C A 1, B R C A 2, P A L B 2$, $A T M$ confer clinical benefit to PARP inhibition across multiple tumor types (26-29). Deficiencies in ATM were hypothesized to be associated with response to PARP inhibition in patients with GC. The phase 3 GOLD study randomized patients with advanced, previously treated GC, to weekly paclitaxel plus or minus olaparib (13). The study failed to improve OS in the overall study population or in patients with ATM-low tumors. Notably, the inclusion criteria for this study required prior therapy with a platinum-containing regimen; however, the time interval between progression after platinum chemotherapy and study enrollment was highly variable. Another open question surrounds whether under-dosing of olaparib to accommodate chemotherapy may have compromised optimal inhibition of PARP. An association between initial response to platinum chemotherapy and subsequent sensitivity to PARP-inhibition is currently not known.

Future studies require defining optimal cutoffs for low ATM expression. Immune checkpoint inhibitors now available for advanced GC pose interesting combinatorial strategies (30,31). Recent preclinical studies have implicated ATM as being required for inducing PD-L1 expression in response to radiation-induced cellular DNA damage (32). Whether deficiency in ATM and its apparent linkage to impairing adaptive immune resistance mechanisms translates into improved outcomes for patients receiving combination of platinum and immune checkpoint agents needs further study. Examining tumor ATM expression from patient specimens in such ongoing trials is of interest.

\section{Acknowledgements}

Funding: This work was supported by the National Institute of Health (5K12CA001727-23 to J Cao) and the Korean Health Technology R\&D Project, Ministry of Health \& Welfare, Republic of Korea (HI14C3418, HI16C1990 to J Lee). Support was also provided by a grant from the 20 by 20 project of Samsung Medical Center (GF01140111 to J Lee).

\section{Footnote}

Conflicts of Interest: SJ Klempner receives research funding (institutional) form LEAP therapeutics and Astellas and has served as a consultant/advisor for Lilly Oncology, Astellas, Boston Biomedical and Foundation Medicine. J Chao receives research funding from Merck (institutional) and has served as a consultant/advisor for Merck, Boston Biomedical, and Five Prime Therapeutics. The remaining authors have no conflicts of interest to declare.

Ethical Statement: The study was approved by Samsung Medical Center (IRB 2015-10-062) and informed consent was taken from all patients.

\section{References}

1. Ferlay J, Soerjomataram I, Dikshit R, et al. Cancer incidence and mortality worldwide: sources, methods and major patterns in GLOBOCAN 2012. Int J Cancer 2015;136:E359-86.

2. Shah MA, Bang YJ, Lordick F, et al. Effect of Fluorouracil, Leucovorin, and Oxaliplatin With or Without Onartuzumab in HER2-Negative, MET-Positive Gastroesophageal Adenocarcinoma: The METGastric Randomized Clinical Trial. JAMA Oncol 2017;3:620-7.

3. Chao J, Lee J, Klempner SJ. Moving molecular subtypes to the clinic in gastric cancer. Transl Cancer Res 2016;5:S25-30.

4. Waddell T, Chau I, Cunningham D, et al. Epirubicin, oxaliplatin, and capecitabine with or without panitumumab for patients with previously untreated advanced oesophagogastric cancer (REAL3): a randomised, openlabel phase 3 trial. Lancet Oncol 2013;14:481-9.

5. Al-Batran SE, Homann N, Pauligk C, et al. Effect of Neoadjuvant Chemotherapy Followed by Surgical Resection on Survival in Patients With Limited Metastatic Gastric or Gastroesophageal Junction Cancer: The AIOFLOT3 Trial. JAMA Oncol 2017;3:1237-44.

6. Bang YJ, Van Cutsem E, Feyereislova A, et al. Trastuzumab in combination with chemotherapy versus chemotherapy alone for treatment of HER2-positive 
advanced gastric or gastro-oesophageal junction cancer (ToGA): a phase 3, open-label, randomised controlled trial. Lancet 2010;376:687-97.

7. O'Connor MJ. Targeting the DNA Damage Response in Cancer. Mol Cell 2015;60:547-60.

8. Matsuoka S, Ballif BA, Smogorzewska A, et al. ATM and ATR substrate analysis reveals extensive protein networks responsive to DNA damage. Science 2007;316:1160-6.

9. Choi M, Kipps T, Kurzrock R. ATM Mutations in Cancer: Therapeutic Implications. Mol Cancer Ther 2016;15:1781-91.

10. Vencken PM, Kriege M, Hoogwerf D, et al. Chemosensitivity and outcome of BRCA1 - and BRCA2associated ovarian cancer patients after first-line chemotherapy compared with sporadic ovarian cancer patients. Ann Oncol 2011;22:1346-52.

11. Kim JW, Im SA, Kim MA, et al. Ataxia-telangiectasiamutated protein expression with microsatellite instability in gastric cancer as prognostic marker. Int J Cancer 2014;134:72-80.

12. Guo X, Shen D, Cheng W, et al. ATM deficiency sensitizes gastric cancer cells to the PARP inhibitior olaparib. Mol Cancer Ther 2009;8: abstr B42.

13. Bang YJ, Xu RH, Chin K, et al. Olaparib in combination with paclitaxel in patients with advanced gastric cancer who have progressed following first-line therapy (GOLD): a double-blind, randomised, placebo-controlled, phase 3 trial. Lancet Oncol 2017;18:1637-51.

14. Lee HE, Kim MA, Lee HS, et al. MET in gastric carcinomas: comparison between protein expression and gene copy number and impact on clinical outcome. Br J Cancer 2012;107:325-33.

15. Kim HS, Kim MA, Hodgson D, et al. Concordance of ATM (ataxia telangiectasia mutated) immunohistochemistry between biopsy or metastatic tumor samples and primary tumors in gastric cancer patients. Pathobiology 2013;80:127-37.

16. Ichikawa $H$, Nagahashi $M$, Shimada $Y$, et al. Actionable gene-based classification toward precision medicine in gastric cancer. Genome Med 2017;9:93.

17. Wang J, Sun Y, Bertagnolli MM. Comparison of gastric cancer survival between Caucasian and Asian patients treated in the United States: results from the Surveillance Epidemiology and End Results (SEER) database. Ann Surg Oncol 2015;22:2965-71.

18. Johnson N, Johnson SF, Yao W, et al. Stabilization of mutant BRCA1 protein confers PARP inhibitor and platinum resistance. Proc Natl Acad Sci U S A
2013;110:17041-6.

19. Yuan SS, Lee SY, Chen G, et al. BRCA2 is required for ionizing radiation-induced assembly of $\operatorname{Rad} 51$ complex in vivo. Cancer Res 1999;59:3547-51.

20. Alsop K, Fereday S, Meldrum C, et al. BRCA mutation frequency and patterns of treatment response in BRCA mutation-positive women with ovarian cancer: a report from the Australian Ovarian Cancer Study Group. J Clin Oncol 2012;30:2654-63.

21. Humeniuk MS, Zhang T, Armstrong AJ. Exploiting DNA damage without repair: The activity of platinum chemotherapy in BRCA-mutated prostate cancers. Cancer 2017;123:3441-4.

22. Byrski T, Dent R, Blecharz P, et al. Results of a phase II open-label, non-randomized trial of cisplatin chemotherapy in patients with BRCA1-positive metastatic breast cancer. Breast Cancer Res 2012;14:R110.

23. Cui Y, Palii SS, Innes CL, et al. Depletion of ATR selectively sensitizes ATM-deficient human mammary epithelial cells to ionizing radiation and DNA-damaging agents. Cell Cycle 2014;13:3541-50.

24. Teng PN, Bateman NW, Darcy KM, et al. Pharmacologic inhibition of ATR and ATM offers clinically important distinctions to enhancing platinum or radiation response in ovarian, endometrial, and cervical cancer cells. Gynecol Oncol 2015;136:554-61.

25. Kass EM, Helgadottir HR, Chen CC, et al. Double-strand break repair by homologous recombination in primary mouse somatic cells requires BRCA1 but not the ATM kinase. Proc Natl Acad Sci U S A 2013;110:5564-9.

26. Bhangoo MS, Costantini C, Clifford BT, et al. Biallelic Deletion of PALB2 Occurs Across Multiple Tumor Types and Suggests Responsiveness to Poly (ADP-ribose) Polymerase Inhibition. JCO Precis Oncol 2017. doi: 10.1200/PO.17.00043.

27. Mirza MR, Monk BJ, Herrstedt J, et al. Niraparib Maintenance Therapy in Platinum-Sensitive, Recurrent Ovarian Cancer. N Engl J Med 2016;375:2154-64.

28. Robson M, Im SA, Senkus E, et al. Olaparib for Metastatic Breast Cancer in Patients with a Germline BRCA Mutation. N Engl J Med 2017;377:523-33.

29. Swisher EM, Lin KK, Oza AM, et al. Rucaparib in relapsed, platinum-sensitive high-grade ovarian carcinoma (ARIEL2 Part 1): an international, multicentre, openlabel, phase 2 trial. Lancet Oncol 2017;18:75-87.

30. Fuchs CS, Doi T, Jang RW, et al. Safety and Efficacy of Pembrolizumab Monotherapy in Patients with Previously Treated Advanced Gastric and Gastroesophageal Junction 
Cancer: Phase 2 Clinical KEYNOTE-059 Trial. JAMA Oncol 2018;4:e180013.

31. Kang YK, Boku N, Satoh T, et al. Nivolumab in patients with advanced gastric or gastro-oesophageal junction cancer refractory to, or intolerant of, at least two previous chemotherapy regimens (ONO-4538-12,

Cite this article as: Klempner SJ, Bhangoo MS, Luu HY, Kim ST, Chao J, Kim KM, Lee J. Low ATM expression and progression-free and overall survival in advanced gastric cancer patients treated with first-line XELOX chemotherapy. J Gastrointest Oncol 2018;9(6):1198-1206. doi: 10.21037/ jgo.2018.09.04
ATTRACTION-2): a randomised, double-blind, placebocontrolled, phase 3 trial. Lancet 2017;390:2461-71.

32. Sato H, Niimi A, Yasuhara T, et al. DNA double-strand break repair pathway regulates PD-L1 expression in cancer cells. Nat Commun 2017;8:1751. 


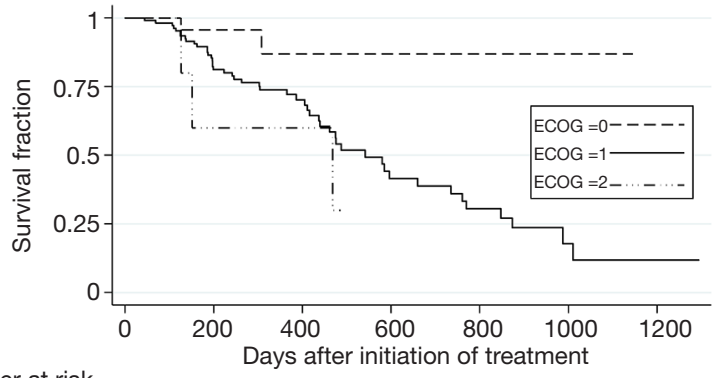

Number at risk

$\begin{array}{lccccccc}\text { ECOG }=0 & 24 & 17 & 7 & 3 & 2 & 1 & 0 \\ \text { ECOG }=1 & 108 & 76 & 37 & 16 & 11 & 3 & 1 \\ \text { ECOG }=2 & 5 & 3 & 2 & 0 & 0 & 0 & 0\end{array}$

Figure S1 Overall survival by ECOG status in 1L XELOX therapy for advanced GC. 1L, first line; GC, gastric cancer; XELOX, capecitabine in combination with oxaliplatin. 